\title{
Determination of Heat and Flow Characteristics in Spray Cooling with Rectangular Finned Heat Sink
}

\author{
Altug Karabey*1, Kenan Yakut ${ }^{2}$ \\ ${ }^{1}$ Ercis Vocational High School, Yuzuncu Yil University, Turkey \\ ${ }^{2}$ Faculty of Engineering, Ataturk University, Turkey \\ ${ }^{*}$ Corresponding author: akarabey@yyu.edu.tr
}

\begin{abstract}
Spray cooling process has many parameters such as extended surface, angle of inclination, effect of gravity, diameter of nozzle, angle of spray, mass flux, geometry of cooled surface, thermal performance and critical heat flux of spray etc. Many effective parameters to carry out the experiments with conventional test methods are both expensive and time consuming. As a solution in these circumstances, Taguchi method, which is one of the modern experimental design and optimization methods and very effective in solving such problems, was used in this study. Taguchi method, as well as being in effective to improve the quality of products, also gives the opportunity to achieve better results with much less experiment. Using Taguchi method, as well as to reach the target value exactly, the sensitivity of the design against uncontrollable factors is reduced to a minimum. Thus, the optimum tolerance range in cost and quality factors is determined. When compared to conventional experimental design methods, Taguchi method has many advantages.

In these experiments with using rectangular pin fin heat sinks, the effects of the longitudinal and lateral distances of the consecutively arranged nozzle or diffuser-like fin pairs, widths of the fins, angle of fins, heights of fins, spraying time, air flow rate, liquid flow rate (ALR, the ratio of air-liquid flow rate) and the ratio of the nozzle-heat sink distance to the nozzle diameter $(\mathrm{h} / \mathrm{d})$ on heat and flow characteristics have been investigated by using Taguchi experimental design method. For this reason, characteristics of flow and heat transfer are considered separately. Nusselt number considered as performance statistic, $L_{27}\left(3^{11}\right)$ orthogonal array has been selected as an experimental design plan for the eleven parameters mentioned above. The Nusselt number was calculated by taking into account the characteristic length of heat sink and the optimized results were found to be fin width of $45 \mathrm{~mm}$, fin angle of $45^{\circ}$, fin height of $15 \mathrm{~mm}$, $x$ direction distance between fins of $20 \mathrm{~mm}$, y direction distance between fins of $20 \mathrm{~mm}$, $x$ direction distance between slices of $15 \mathrm{~mm}$, y direction distances between slices of $20 \mathrm{~mm}$, air flow rate of $10^{-3} \mathrm{~m}^{3} / \mathrm{s}$, liquid flow rate of $5,83^{*} 10^{-6} \mathrm{~m}^{3} / \mathrm{s}$, spraying time of $5 \mathrm{~s}$ and the ratio of the nozzle-heat sink distance to the nozzle diameter $(\mathrm{h} / \mathrm{d})$ of 667 .
\end{abstract}

\section{Keywords}

Spray cooling, Taguchi Methods, heat sink.

\section{Introduction}

Atomization is used to transfer energy from wide surface areas in low temperatures throughout cooling applications. From the outlet of an atomizer, an unsteady liquid mass performs a fragmentation process respectively in the order of layer formation, ligament formation, and droplet formation. The velocity and diameter rates of the droplets to compose spray are important. When the droplets with a certain speed and diameter range are grouped, the droplet speed and diameter range occur, and these two ranges are fundamental to characterizing the spray.

Effective energy transfer is ensured with spray cooling, a technology characterized by high heat transfer, heat convection homogeneity, and a low droplet impact speed, which are explored for engineering applications today. Having gained momentum especially in the last 20 years, studies on the atomization of liquids have been used widely in industry. The cooling of metal surfaces and electronic components, drying operations, humidification, washing, watering and fire extinguishing operations, pulverized fuel injection systems, surface coating and spray painting operations, agricultural disinfection, drug manufacturing, and spray medicine mechanisms taken orally and through the nose can be cited as applications of atomization. Because atomization has an extensive area of use, empirical studies are required so spray parameters can be analyzed qualitatively and quantitatively [10]. Analyzing the impacts of all determined parameters on the performances of thermal systems and system elements is almost impossible in terms of both time and testing costs when using the strategy of making tests by changing a parameter at any time. By not changing a parameter at every turn, it is possible to ensure a significant decline in the number of experiments by using only those selected carefully from full factorial designs. With this testing method, randomization is used to eliminate the impact of heterogeneity on testing methods. Thanks to such a course of 
action, it is possible to reduce the adverse effects of unexpected changes (i.e., ambient temperature, humidity, pressure, etc.) on uncontrolled factors [11].

With the method first developed by Japanese engineer Dr. Taguchi, it is possible to minimize the variations in product and process by selecting the most appropriate levels of controllable factors against the factors that create these variations and that are uncontrolled. In his testing strategy, Taguchi uses high fractional experiments, including orthogonal sequences, to specify optimum values of controllable factors. With the analysis of the data obtained after the experiments, whether the anticipated result would be achieved is controlled for by conducting confirmation experiments in optimum conditions determined thereof. After the performance values and signal-tonoise ratio-obtained following the experiments-are analyzed and this classification is made, variation is reduced with the help of control factors, and average targeted values are achieved with the help of correction factors. Furthermore, the most suitable and economic values of inefficient factors are selected.

In Taguchi's testing strategy, high fractional experiments, including orthogonal sequences, are used to specify the optimum values of controllable factors. With the analysis of the data obtained after the experiments, whether the anticipated result would be achieved is controlled for by conducting confirmation experiments in optimum conditions determined thereof [11]. Phadke [5], states Taguchi developed 18 different orthogonal sequences. Taguchi also developed linear graphics and triple charts to be based on to ensure the placement of factors and the combined effects in these sequences. In many problems, one of the standard orthogonal sequences may be used as an experimental design directly. By making use of linear graphics, triple charts, and similar instruments in some cases, an experimental design suitable for the problem may be developed with partial arrangements in standard orthogonal sequences. In other words, simplicity or perfect flexibility is ensured in planning multi-factor or multi-level experiments.

After the performance values and signal-to-noise ratio-obtained following the experiments—are analyzed and this classification is made, variation is reduced with the help of control factors, and average targeted values are achieved with the help of correction factors. Furthermore, the most suitable and economic values of inefficient factors are selected [1].

In this study, the impact of 11 parameters with three levels on the optimum operation conditions of a heat sink was determined by means of Taguchi's experimental design method, which was applied to spray cooling. Positive and negative aspects of the method were discussed in studies regarding the increase in the heat transfer in heat exchangers.

\section{Material and methods}

Experimental parameters and plan

In this study, the optimum parameters of heat sink in Figure 1, which are sequenced in the form of a longitudinal and lateral distances were determined in terms of heat transfer using Taguchi's experimental design technique.

Parameters having an impact on heat transfer were selected as the fin width, fin angle, fin height, horizontal distance between fins, vertical distance between fins, horizontal distance between slices, vertical distance between slices, rate of nozzle-heat sink distance to nozzle diameter, air flow, water flow, and spraying time. As well, the $L_{27}\left(3^{11}\right)$ orthogonal array was selected as the experimental design. Experiments were performed in the spray cooling testing apparatus indicated in Figure 2. This study used a heat sink $300 \times 300 \mathrm{~mm}$ in size with a testing pin fin on the heat sink and $\mathrm{h} / \mathrm{d}$ distance, which is adjustable, on which an air-supported atomizer $1.2 \mathrm{~mm}$ in diameter can be mounted, along with a flow meter and manometer for air-water fluids. Air and water were preferred as fluids in the system. A 15-liter water/oil bath that operates in a closed system was placed near the testing area so experiments could be performed at constant temperatures. With the experimental setup set (Figure 2), such parameters as nozzle outlet speed of spray, droplet diameter, distribution of spray droplets in the testing area, and amount of surface contact were obtained with the help of a charge coupled device (CDD) camera and stroboscope, and the temperature distributions were obtained via thermal cameras.

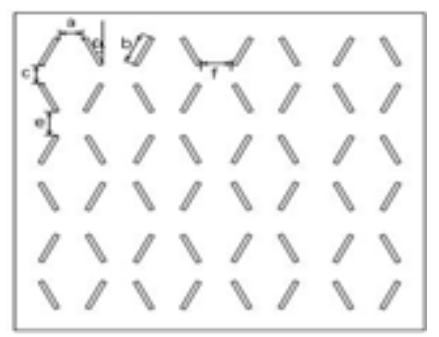

Figure 1. Geometrical definitions of the tested models. 


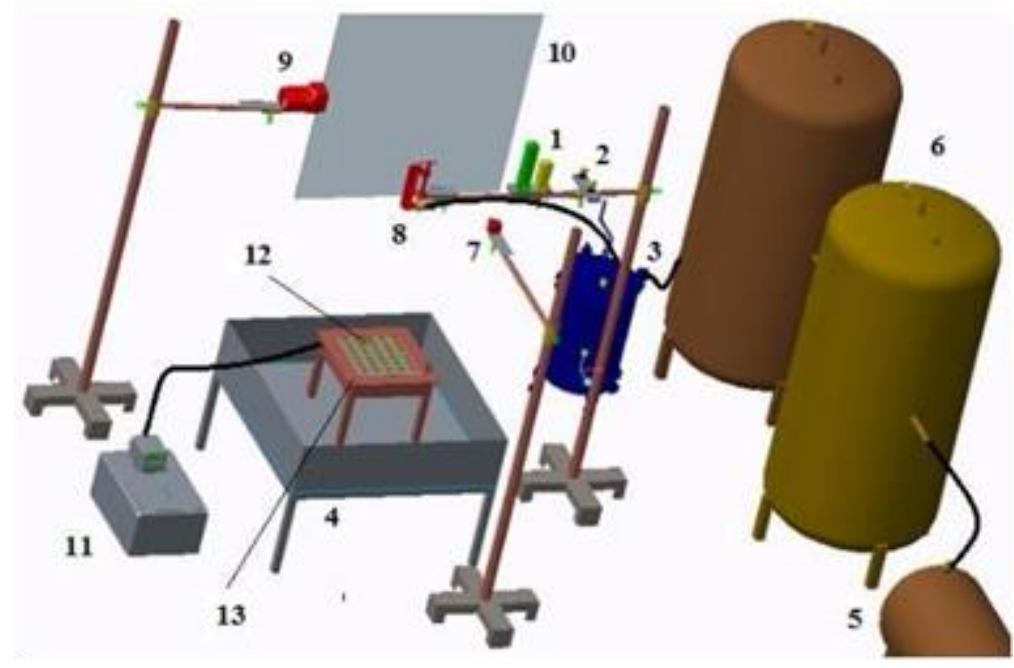

Figure 2. A schematic display of the experimental setup:

(1) Flow meter, (2) manometer, (3) pressurized water tank, (4) reservoir, (5) compressor, (6) air tank, (7) CCD camera, (8) nozzle, (9) stroboscope, (10) background, (11) water/oil bath, (12) test-section, (13) reactor.

Table 1 shows the controllable parameters selected in consideration of the fact that they are likely to affect the characteristics of heat transfer and the values of these parameters and that were analyzed in the tests.

Table 1. Parameters and their values studied in the experiments.

\begin{tabular}{|c|c|c|c|c|}
\hline & & \multicolumn{3}{|l|}{ Level } \\
\hline \multicolumn{2}{|c|}{ Parameter } & 1 & 2 & 3 \\
\hline $\mathrm{A}$ & $\mathrm{h} / \mathrm{d}$ ratio & 166.6 & 333.3 & 666.6 \\
\hline B & Fin width, b [mm] & 15 & 30 & 45 \\
\hline C & Angle of fin, $\alpha$ [degree] & 15 & 30 & 45 \\
\hline D & Fin height, $\mathrm{H}[\mathrm{mm}]$ & 15 & 25 & 35 \\
\hline$E$ & Horizontal distance between fins, a [mm] & 10 & 15 & 20 \\
\hline $\mathrm{F}$ & Vertical distance between fins, $\mathrm{c}[\mathrm{mm}]$ & 10 & 15 & 20 \\
\hline G & Vertical distance between slices, e [mm] & 10 & 15 & 20 \\
\hline $\mathrm{H}$ & Horizontal distance between slices, $f$ [mm] & 10 & 15 & 20 \\
\hline I & Air flow, $Q_{a}\left[\mathrm{~m}^{3} / \mathrm{h}\right]$ & 2.1 & 2.9 & 3.6 \\
\hline $\mathrm{J}$ & Water flow, Q। $\left[\mathrm{m}^{3} / \mathrm{h}\right]$ & 0.012 & 0.021 & 0.03 \\
\hline $\mathrm{K}$ & Spraying time, $\mathrm{T}[\mathrm{s}]$ & 5 & 10 & 15 \\
\hline
\end{tabular}

Table 1 shows that all parameters were selected to have three levels, and the $L_{27}\left(3^{11}\right)$ orthogonal array $(O A)$ experimental design method was selected accordingly as the experimental plan [5].

Experimental plan shows that only 54 experiments were conducted with 27 heat sink unit using the $\operatorname{L}_{27}\left(3^{11}\right)$ experimental design and Taguchi's experimental design instead of conducting $3^{11}=177,147$ experiments with a full factorial experimental design from the classical testing design methods. Each experiment was repeated twice at different times so the effects of deforming and the random factors in the experiments could be observed. "Performance statistics" was selected as the optimization criteria. Performance values in terms of the Nusselt number were calculated to be able to observe the impacts of the parameters on the optimization criteria. The Nusselt number was calculated based on nozzle diameter in consideration of the convection heat transfer coefficient, which is calculated based on the total area of the heat transition surface, and a general optimum was found. The "the bigger the better" performance statistic was used for Nusselt numbers.

$$
Z_{B}=-10 \log \left(\frac{1}{n} \sum_{i=1}^{n} \frac{1}{Y_{i}^{2}}\right)
$$

Kackar [6], states that many (more than 60) performance statistics likely to be used based on the analysed problem were developed. The following performance statistic, was developed for the "the bigger the better" status and is one of the alternatives that could be selected for the optimization criteria, where $Z_{B}$ and $Z_{K}$ indicate performance 
statistics, $n$ indicates the number of repetitions made in an experimental combination, and $Y_{i}$ shows the performance value of the test.

In the Taguchi method, the experiment corresponding to the optimum working conditions might not have been undertaken during the whole period of experimentation. In such cases, the performance value corresponding to optimum working conditions can be predicted by utilizing the balanced characteristic of the OA. For this aim, the additive model may be used [7]

$Y_{i}=\mu+X_{i}+e_{i}$

where indicates the general average performance value, $X_{i}$ indicates the fixed impact of the parameter-level combination in the test, and $e_{i}$ indicates the random fault in the experiment. As the equation is a point estimation, calculated using experimental data, a confidence interval must be calculated to determine whether this value is significant. A confidence interval at the selected error level may be calculated with the help of the following equation [8],

$Y_{i} \pm \sqrt{F_{\alpha ; 1, D F_{M S e}} * M S e *\left(\frac{1+m}{N}+\frac{1}{n_{r}}\right)}$

where $F$ refers to chart value, $\alpha$ indicates the error level, DF ${ }_{M S e}$ indicates the total degree of freedom for the average of the error squares, $m$ refers to the total degree of freedom for the parameters used in estimating optimum working conditions, $N$ refers to the total number of tests/experiments, and $n_{i}$ indicates the number of repetitions in the confirmation experiment. If the experimental results are in terms of percentage (\%), an omega conversion of percentage values is performed with the help of the following equation before calculating Equations (3) and (4). After, the values of concern are determined by applying a reverse-conversion with the help of the same equation [9],

$\Omega(d b)=-10 \log \left(\frac{1}{P}-1\right)$

where $\Omega(d b)$ indicates the decibel value, which was found with the omega conversion of the percentage value, and $p$ indicates the percentage value of the experimentally obtained product.

Calculating the heat transfer coefficient

For this study, the Bi number was found to be significantly low for the aluminum heat sinks with rectangular fins at a constant surface temperature in the non-boiling regime, and it was decided to use a total mass approach. Accordingly, the amount of heat generated on the aluminum surface was calculated with the following equations,

$Q_{\text {total }}=Q_{\text {konv }}+Q_{\text {rad }}+Q_{\text {vap }}$

and

$Q=m_{A l} C_{p} \Delta T$

Where $\Delta T$ refers to average surface temperatures of the surface before and after spray.

Tahat et al. [2] reported that radiative heat-transfer rate from a pin-fin depends on: (i) the temperature of its radiating surface; (ii) the temperature distribution of its surroundings; (iii) the emissivities of the fin, base and environment; and (iv) the shielding effect of adjacent fins. The radiating surface temperature and the shielding effect determine the grey-body shape-factor $F$.

The total steady-state rate of radiative heat-transfer can be evaluated from

$Q_{\text {rad }}=F A_{S}\left(T_{S}^{4}-T_{a}^{4}\right)$

In similar studies Tahat et al. [2], Tahat et al. [3], El-sayed et al. [4], etc., reported that the total radiative heat-losses from a similar test surface would be about $0.5 \%$ of the total heat-input. Also the base plate and fins were of aluminum; their surfaces being highly matted using black spray painting and so of low emissivity. Therefore, the radiative heat loss could be neglected. Using these findings, together with the fact that the test section was well insulated and the readings of the thermojunction placed at the outer surface of the heating section was nearly equal to the ambient temperature, then one could assume with some confidence that the last two terms of Eq. (6) may be ignored. Then, the Eq. (6) reduces to 
$Q_{\text {total }}=Q_{\text {Conv }}$

As for the heat transfer coefficient for the spray, sent via the air-supported nozzle, it was identified as follows;

$h_{s p}=\frac{\left(Q-Q_{r a d}-Q_{b u h .}\right)}{A_{y}\left(T_{y}-T_{s p}\right)}$

When the studies in the literature are reviewed, both the projection area and total heat transfer area are used as the heat transfer surface area. In this study, the total heat transfer area was used. This area refers to the total heat transfer surface area created by the fins and base plate, and it can be identified as follows:

$$
A_{s}=W L+N\left[\left(2 b h_{k}\right)+\left(2 t h_{k}\right)\right]
$$

The Nusselt number was considered a performance statistic; it was calculated with the following equation based on the inner diameter of the nozzle while calculating the Nusselt number:

$N u=\frac{h_{s p} D_{h}}{k}$

In addition, the heat thrown due to vaporization was calculated for several spraying times, according to the change in the relative humidity on the surface. This value was determined to be $14.72 \%, 16.92 \%$, and $19.31 \%$, respectively, for $5 \mathrm{~s}, 10 \mathrm{~s}$, and $15 \mathrm{~s}$ of spraying. The forces playing a role in the fragmentation of unsteady liquid masses may be identified as inertia forces, surface tension, and viscous forces. This is why pure numbers to characterize these kinds of flows consist of different combinations of these three different forces. The first that comes to mind among the pure numbers used to characterize any forced flow problem is the Reynolds number, which is known as the rate of inertia forces to viscous forces:

$\operatorname{Re}=\frac{D_{h} U_{\text {ort }}}{v}$

In the equation, thermo-physical specifications and the average speed Uort of the liquid in the nozzle cross-section were determined in consideration of the segment of the fluid in the nozzle outlet.

\section{Results and discussion}

Following the tests and measurements, the collected data were analyzed using the ANOVA ${ }^{\mathrm{TM}}$ packet program to determine the impact of each parameter on the performance statistic. The average surface temperatures were calculated for all experiments with a thermal camera before and after the spraying operation. An example, performed for Experiment-4, is included in Figure 3 below.

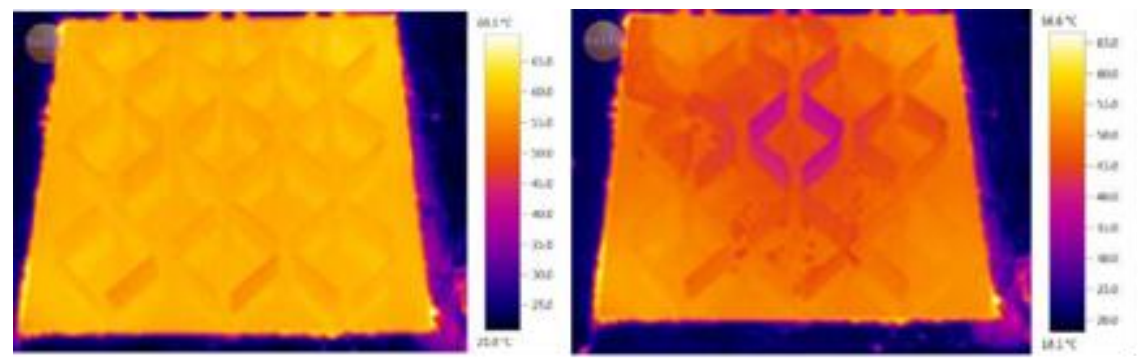

Figure 3. Thermal camera images.

The impact of each parameter on optimization was calculated and results are presented in Figure 4. The order of graphics was determined in accordance with the degree of the impact of the parameters on the performance statistic. In the graphics, the numerical value of the maximum point indicates the best value of the respective parameter, and the numerical value of the minimum point indicates the worst value of the respective parameter. In the effect graphic to the Nusselt number (Figure 5), the maximum points for all parameters indicate the optimum levels of the parameter selected in the experimental study conducted thereof. 


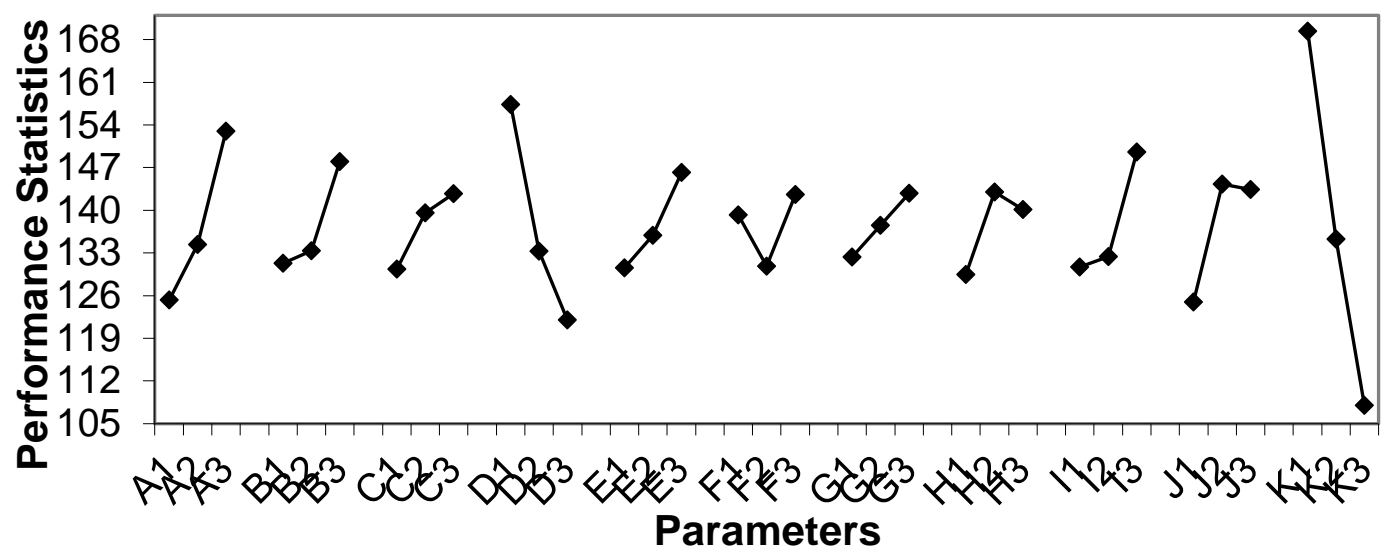

Figure 4. The effect of each parameter on the Nusselt number.

It seems difficult and complex to understand experimental results from the graphics at first glance. If the procedure is reviewed with an example, the fin width and variation in the performance statistic B are indicated in Table 1. The fin width is $15 \mathrm{~mm}$ at the first point, and this corresponds to the first level of the parameter (see Table 1). Experiments corresponding to Level 1 can be found in experimental plan. Test numbers from experimental plan are 1, 2, 3, 10, $11,12,19,20,21$. The performance statistic value is the average of the data obtained from these tests. In Figure 4 , the experimental conditions for the second data point in th plan and the average of the experiments are indicated with 2 in experimental plan (i.e., 4, 5, 6, 13, 14, 15, 22, 23, and 24). The numerical value of the maximum point in each graphic gives the best value of the respective parameter. These values are included in Table 3 for each parameter, referring to the optimum value of each parameter under experimental conditions. Besides, performance values of the combinations corresponding to optimum conditions, estimated with the help of Equation (2) in Table 3 and the confidence interval at a 5\% error level of these estimations, were calculated with the help of Equation (4), and they are included in Table 3. Moreover, confirmation experiments were conducted under the determined optimum conditions to be able to test the accuracy of these estimations, and the results are presented in the "Real" line. Because the performance values obtained in confirmation experiments are within the calculated confidence interval, it can be stated that experimental results are acceptable at a $5 \%$ error level.

Table 3. Optimum conditions and performance values for tested models.

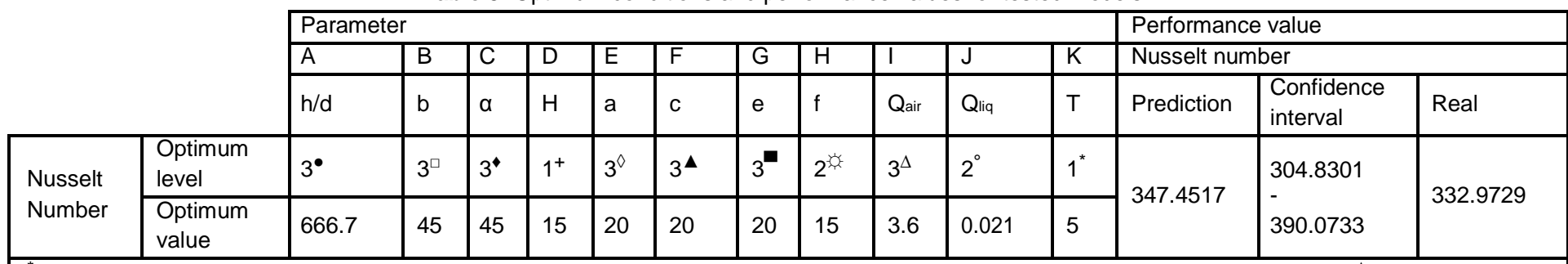

*: The first effective parameter ${ }^{+}$: The second effective parameter $\bullet:$ The third effective parameter ${ }^{\circ}:$ The fourth effective parameter ${ }^{\Delta}:$ The fifth effective parameter ${ }^{\square}$ : The sixth effective parameter ${ }^{\diamond}$ : The seventh effective parameter ${ }^{\star}$ : The eighth effective parameter $\downarrow$ : The ninth effective parameter

$\boldsymbol{\Delta}:$ The tenth effective parameter

Contribution percentages of the impacts of each parameter on the selected performance characteristic are indicated in Figures 5. Contribution percentage indicates the impact of the respective parameter on the performance statistic, and it is calculated as follows:

$\mathrm{KY}=([$ Squares total-(Degree of freedom*Total errors) $]) /($ Total of squares $)$ 


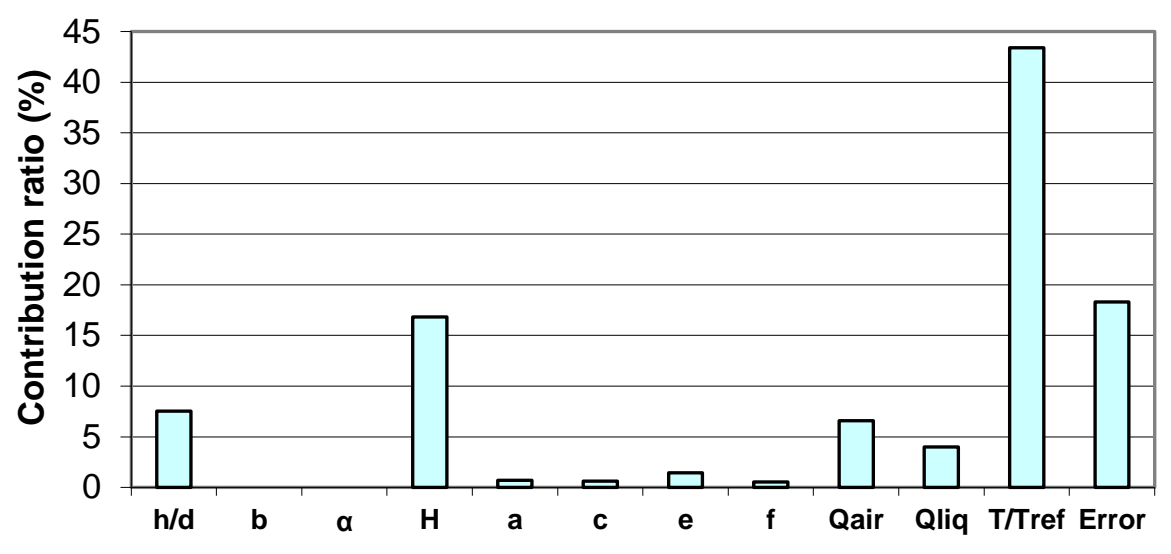

Figure 5. Contribution ratio of each factor to the Nusselt number.

The parameters having the greatest impact on the Nusselt number are, respectively, spraying time ( $T)$, fin height $(\mathrm{H})$, the rate of nozzle-heat sink distance to nozzle diameter (h/d), water flow (Qliq), air flow (Qair), fin width (b), horizontal distance between fins (a), horizontal distance between slices (f), fin angle $(\alpha)$, vertical distance between fins (c), and vertical distance between slices (e). It was determined that the parameter having the greatest effect on the Nusselt number is spraying time. Based on the surface geometry, the highest Nusselt number was calculated as 234.4 when the spraying time was $5 \mathrm{~s}$. This indicates there is no additional spraying operation to achieve an optimum cooling load. It was determined that fin height is the parameter having the second-greatest effect on the Nusselt number. In cases where the fin height is $15 \mathrm{~mm}$, the highest Nusselt number calculated was 234.4 based on surface geometry. While the Nusselt numbers were being calculated, the average heat convection coefficient was determined according to the total heat transition area. In the equation, the heat transition surface area from the fins was also considered, and the heat convection coefficient was reduced, as the heat transition area would increase in the event that fin height increases.

The parameter having the third-greatest effect on the Nusselt number was the rate of the nozzle-heat sink distance to nozzle diameter $(\mathrm{h} / \mathrm{d})$. The highest Nusselt number calculated was 234.4 based on the surface geometry at the point where the $\mathrm{h} / \mathrm{d}$ ratio is a maximum 666.7 .

\section{Conclusions}

This experimental study was conducted to determine the heat transfer and flow characteristics of a spray cooling application for a heat sink consisting of rectangular fins with a longitudinal and lateral geometry. Performance criteria regarding the Nusselt number were calculated to be able to observe the impacts of parameters on the optimization criteria by making use of the Taguchi experimental design method and the $L_{27}\left(3^{11}\right)$ experimental design, and the optimum heat sink geometry was obtained. Results for a general optimum heat sink, obtained based on the Nusselt number, were determined to include a $15-\mathrm{mm}$ fin height, $45-\mathrm{mm}$ fin width, $45^{\circ}$ fin angle, $20-\mathrm{mm}$ distance between fins, 20-mm vertical distance between fins, 20-mm horizontal distance between slices, 15-mm vertical distance between slices, 666.7 rate of nozzle-heat sink distance to nozzle diameter, $3.6-\mathrm{m}^{3} / \mathrm{h}$ air flow, $0.021-\mathrm{m}^{3} / \mathrm{h}$ water flow, and 5-s spraying time. The parameters having the greatest impact on the Nusselt number are, respectively, spraying time $(T)$, fin height $(H)$, the ratio of nozzle-heat sink distance to nozzle diameter $(\mathrm{h} / \mathrm{d})$, water flow (Qliq), air flow (Qair), fin width (b), horizontal distance between fins (a), horizontal distance between slices (f), fin angle ( $\alpha$ ), vertical distance between fins, (c) and vertical distance between slices (e). It was determined that spraying time is the parameter having the greatest effect on the Nusselt number. Studies to determine spray flow characteristics are rather complex. Therefore, it is essential to measure via a laser that enables a detailed analysis of the flow structure (PIV). Florinert liquids, such as FC-72 and FC-87, can be used in spray cooling as an operation liquid. The heat transfer process must be reviewed by adding surface-active agents. This study was performed below the boiling point, but studies hereafter may be conducted above the boiling point or at the Leidenfrost temperature. It is essential to research air-supported nozzles, which are also used in this study to design different nozzle geometries. Heat transfer and the flow process can be analyzed mathematically using such software as ANSYS-FLUENT.

\section{Acknowledgements}

This research was supported as project (BAP-2013/103) of the Research Fund of Ataturk University. 


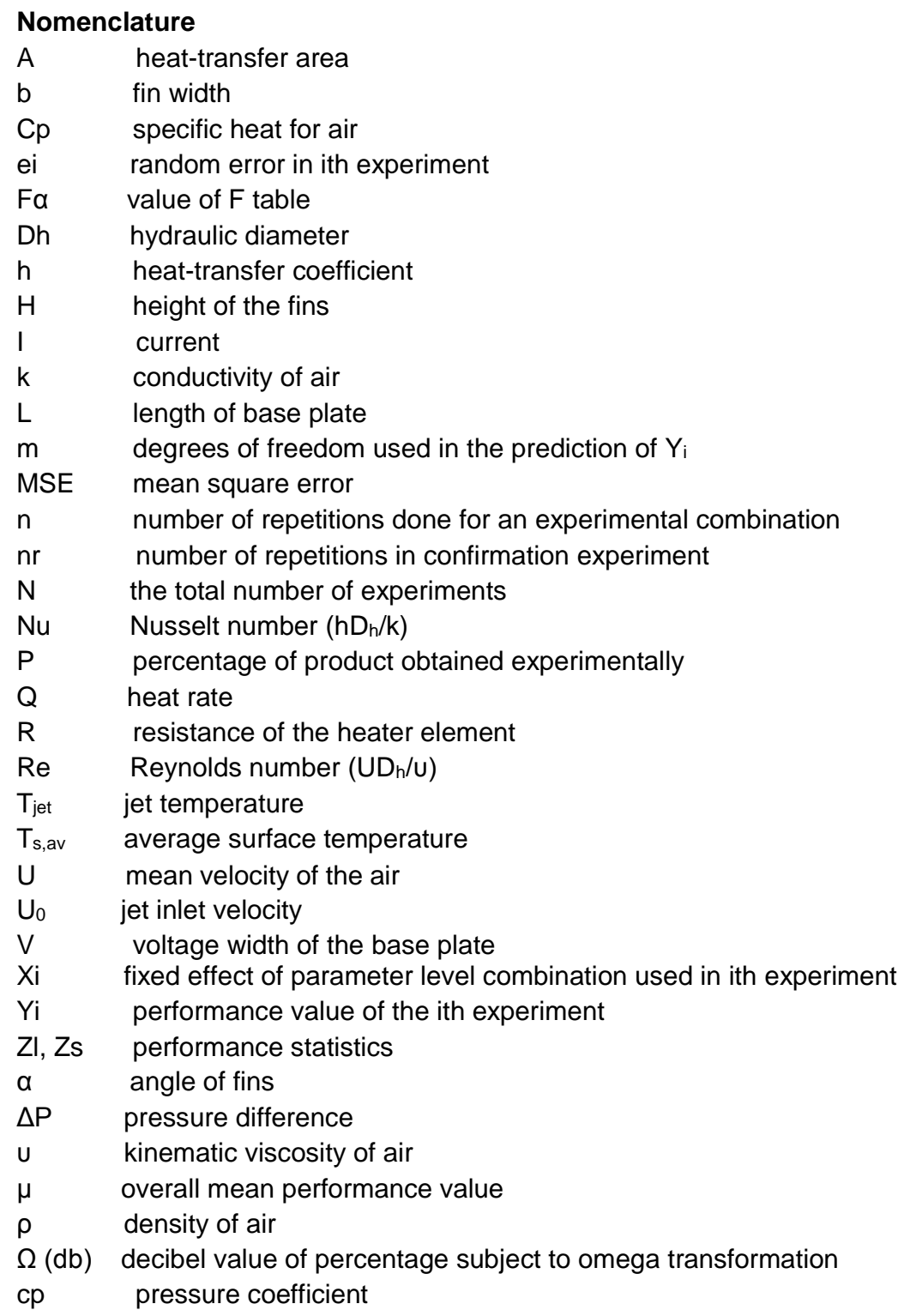

\section{References}

[1] Sahin B., Yakut K., Kotcioglu I., Celik C. Optimum design parameters of a heat exchanger. Applied Energy 2005; 82:90-106.

[2] Tahat MA, Babus_Haq RF, Probert SD. Forced steady-state convection from pin-fin arrays. Appl Energy 1994;48:335-51.

[3] Tahat M, Kodah ZH, Jarrah BA, Probert SD. Heat transfers from pin-fin arrays experiencing forced convection. Appl Energy 2000;67/4:419-42.

[4] El-Sayed SA, Mohamed MS, Abdel-latif AM, Abouda AE. Investigation of turbulent heat transfer and fluid flow in longitudinal rectangular-fin arrays of different geometries and shrouded fin array. Exp Therm Fluid Sci 2002;26:879900.

[5] Phadke MS. Quality engineering using robust design. Englewood Cliffs (NJ): Prentice-Hall; 1989.

[6] Kackar RN. Off-line quality control, parameter design and Taguchi methods. J Qual Technol 1985;17:176-209.

[7] Phadke MS, Kackar RN, Speeney DV, Grieco MJ. Off-line quality control in integrated fabrication using an experimental design. Bell Sys Tech J 1983;62/5:1273-309.

[8] Ross PJ. Taguchi techniques for quality engineering. Singapore: McGraw-Hill; 1989.

[9] Taguchi G. System of experimental design, quality resources. New York: International Publications; 1987.

[10] Karabey A. Determination of spray characteristics of impingement jet for heat exchanger. PhD Thesis [In Turkish], Ataturk University; 2016.

[11] Celik, C. (1996). Guided experimental techniques in design. Industrial Engineering, 7(6), 14-20. 